\title{
Aberrant sodium influx causes cardiomyopathy and atrial fibrillation in mice
}

\author{
Elaine Wan, ${ }^{1}$ Jeffrey Abrams, ${ }^{1}$ Richard L. Weinberg, ${ }^{1}$ Alexander N. Katchman, ${ }^{1}$ Joseph Bayne, ${ }^{1}$ Sergey I. Zakharov, ${ }^{1}$ Lin Yang, \\ John P. Morrow, ${ }^{1}$ Hasan Garan, ${ }^{1}$ and Steven 0. Marx ${ }^{1,2}$
}

'Division of Cardiology, Department of Medicine, and ²Department of Pharmacology, College of Physicians and Surgeons, Columbia University, New York, New York, USA

\begin{abstract}
Increased sodium influx via incomplete inactivation of the major cardiac sodium channel $\mathrm{Na}_{\mathrm{v}} 1.5$ is correlated with an increased incidence of atrial fibrillation (AF) in humans. Here, we sought to determine whether increased sodium entry is sufficient to cause the structural and electrophysiological perturbations that are required to initiate and sustain AF. We used mice expressing a human $\mathrm{Na}_{v} 1.5$ variant with a mutation in the anesthetic-binding site (F1759A- $\left.\mathrm{Na}_{\mathrm{v}} 1.5\right)$ and demonstrated that incomplete $\mathrm{Na}^{+}$channel inactivation is sufficient to drive structural alterations, including atrial and ventricular enlargement, myofibril disarray, fibrosis and mitochondrial injury, and electrophysiological dysfunctions that together lead to spontaneous and prolonged episodes of AF in these mice. Using this model, we determined that the increase in a persistent sodium current causes heterogeneously prolonged action potential duration and rotors, as well as wave and wavelets in the atria, and thereby mimics mechanistic theories that have been proposed for AF in humans. Acute inhibition of the sodium-calcium exchanger, which targets the downstream effects of enhanced sodium entry, markedly reduced the burden of AF and ventricular arrhythmias in this model, suggesting a potential therapeutic approach for AF. Together, our results indicate that these mice will be important for assessing the cellular mechanisms and potential effectiveness of antiarrhythmic therapies.
\end{abstract}

\section{Introduction}

Atrial fibrillation (AF), estimated to affect about $6 \%$ of Americans who are 65 years of age and older, doubles the risk of death and accounts for $15 \%-20 \%$ of all strokes. It is the most frequent sustained arrhythmia observed in clinical practice. Treatments for AF are limited by the relatively low efficacy of pharmaceuticals and radiofrequency ablation/surgery and the high rates of recurrence (1).

Predisposing contributors to AF are systemic and cardiac disorders, which may induce atrial enlargement, fibrosis, and electrical abnormalities (2). In $10 \%-20 \%$ of cases, however, AF is primarily an electrical disorder, not associated with underlying systemic and cardiac disorders (lone AF). There is also an important component of genetic susceptibility, shown by recent genome-wide association studies and identification of relatively rare mutants in $\mathrm{K}^{+}$and $\mathrm{Na}^{+}$channels and ryanodine receptors (3-5). Two conceptual mechanisms for AF have been proposed: (i) shortening of the atrial effective refractory period, thereby enabling reentry wavelets, and (ii) action potential duration (APD) prolongation, increasing the likelihood of early afterdepolarizations. Focal electrical abnormalities within the myocardial sleeves of pulmonary veins - due to increased automaticity, early after-depolarizations, or delayed after-depolarizations - are the primary causes of paroxysmal AF in humans $(3,6)$.

Authorship note: Elaine Wan, Jeffrey Abrams, and Richard L. Weinberg contributed equally to this work and are co-first authors.

Conflict of interest: The authors have declared that no conflict of interest exists. Submitted: September 15, 2015; Accepted: October 15, 2015.

Reference information: J Clin Invest. 2016;126(1):112-122. doi:10.1172/JCI84669.
The $\mathrm{Na}^{+}$current underlies initiation of the cardiac AP and plays a prominent role in $\mathrm{Na}^{+}$homeostasis by loading cardiomyocytes with $\mathrm{Na}^{+}$ions (7). After opening briefly during the upstroke of the $\mathrm{AP}$, individual $\mathrm{Na}^{+}$channels usually inactivate and remain inactivated until repolarization is completed. $\mathrm{Na}^{+}$channel openings after phase 0 create a small late or persistent current that lasts throughout the plateau phase of the cardiac AP. During the AP plateau, membrane resistance is high and even a modest increase of an inward current can cause AP prolongation. $\mathrm{Na}^{+}$channel mutations - pathological conditions such as heart failure, hypoxia, inflammation, oxidative stress, and pharmacological agents - can either delay or destabilize $\mathrm{Na}^{+}$channel inactivation, thereby increasing persistent current (8). Many of these conditions are associated with an increased incidence of AF. In humans, a role for enhanced $\mathrm{Na}^{+}$ influx in the pathogenesis of AF has been speculated, based upon gain-of-function SCN5A genetic variants in lone AF patients (9), increased incidence of AF in LQT3 patients (10), and increased persistent $\mathrm{Na}^{+}$current in patients with permanent $\mathrm{AF}$ (11).

In many diseases, the availability of a mouse model has led to important molecular insights, in part because of the ease of genetic engineering and the large number of preexisting genetically altered mice for cross-breeding. Studies of AF, in contrast, have been hindered by the lack of a mouse model that accurately recapitulates the spontaneous initiation and prolonged periods of AF observed in humans. Most studies use nonphysiological methods, including high-frequency burst pacing, to induce very short episodes in mice; these episodes typically last several seconds in mice, compared with hours, days, or longer for humans .

We generated transgenic (TG) mice with doxycycline-inducible and titratable cardiac-specific expression of FLAG epitope-tagged 
human $\mathrm{Na}_{\mathrm{v}} 1.5$ with a mutation (F1759A) in the local anesthetic binding site (12), which causes window current and persistent $\mathrm{Na}^{+}$current. We found that 2 founder lines with doxycycline-independent low expression of the mutant $\mathrm{Na}^{+}$channels had the phenotype of atrial enlargement; cardiomyopathy; frequent, relatively long episodes of spontaneous AF; and nonsustained polymorphic ventricular tachycardia (VT), observed as early as 5 weeks of age. These mice phenocopied gain-of-function human SCN5A mutations that have been implicated in dilated cardiomyopathy and hypertrophy, and arrhythmias such as long QT syndrome, torsade de pointes, and AF (13). The spontaneous nature and prolonged periods of the atrial arrhythmias enabled the exploration of mechanisms by which dysfunctional $\mathrm{Na}^{+}$channel inactivation causes cardiomyopathy and arrhythmias. A new therapeutic approach for AF was identified by pharmacologically targeting the downstream effects of enhanced $\mathrm{Na}^{+}$entry.

\section{Results}

Expression of F1759A-Na 1.5 in atria and ventricles. A modified $\alpha$-myosin heavy chain $(\alpha-\mathrm{MHC})$ promoter was used as the responder (14), and after these mice were crossed with $\alpha$-MHC reverse tetracycline-controlled transactivator protein (rtTA) mice (15), double TG (dTG) mice with doxycycline-regulated cardiac-specific expression of FLAG-F1759A-Na 1.5 were created (Figure 1A). Four lines of FLAG-F1759A-Na 1.5 were initially established, but 2 distinct founder lines of mice were of particular interest to us because offspring with both the F1759A and rtTA transgenes developed a cardiomyopathy and spontaneous prolonged periods of $\mathrm{AF}$ in the absence of doxycycline treatment. Littermate mice with single transgenes, either rtTApositive or F1759A-positive, were indistinguishable from each other and from non-TG (NTG) mice, in terms of the lack of a cardiac phenotype and F1759A transgene expression, and were therefore used interchangeably as the littermate control group. Thus, random insertion of the Nav1.5 gene could not be the cause of the phenotypes, including AF, because the F1759A-Na 1.5 mice without rtTA failed to demonstrate any phenotype.

Expression of the FLAG-F1759A-Na 1.5 channels in the atria and ventricles assessed using an anti-FLAG antibody was dependent upon cardiac-specific coexpression of rtTA and did not require doxycycline administration (Figure 1, B-E). Naturally, doxycycline further increased expression, but this was unnecessary for our studies, as a unique cardiac phenotype of a cardiomyopathy and spontaneous prolonged episodes of $\mathrm{AF}$ was apparent in the dTG mice in the absence of doxycycline but never in the single TG or NTG mice. Despite an approximate 2-fold increase in the amount of total (TG human + native) $\mathrm{Na}_{\mathrm{v}} 1.5$ transcript in the dTG compared with NTG or single TG littermate mice (Figure 1B), the combined expression of native and $\mathrm{TG} \mathrm{Na}_{\mathrm{v}} 1.5$ protein was nearly equivalent, assessed semiquantitatively with an anti- $\mathrm{Na}_{\mathrm{v}} 1.5$ antibody that recognizes both mouse and human $\mathrm{Na}_{\mathrm{v}} 1.5$ (Figure 1 , $\mathrm{D}$ and E), and quantitatively using cellular electrophysiology. The mean peak $\mathrm{Na}_{\mathrm{v}} 1.5$ current in isolated atrial and ventricular cells, determined with $5 \mathrm{mM} \mathrm{Na}^{+}$rather than physiologic $\mathrm{Na}^{+}$in the extracellular solution to mitigate voltage clamp errors, was not significantly different in F1759A-dTG mice compared with control littermates (Figure 2, A and B, insets, and Figure 2C). The relatively low protein expression of FLAG-F1759A-Na 1.5 in both atrial and ventricular cardiomyocytes in the absence of doxycycline is likely due to a low basal level of rtTA protein binding to the Tet operator sequences in the absence of doxycycline, thereby driving transcription in some cells (15).

The F1759A substitution markedly diminishes use-dependent lidocaine block (16), enabling the differentiation of the electrophysiological profiles of endogenous and mutant-TG $\mathrm{Na}^{+}$channels in cardiomyocytes. We selected $3 \mathrm{mM}$ as the optimal concentration of lidocaine based upon $96 \% \pm 0.5 \%$ block in WT Na 1.5 and $65.2 \% \pm 1.6 \%$ block in heterologously expressed F1759A-Na 1.5 . Greater than $5 \%$ of peak $\mathrm{Na}^{+}$current remained after exposure to 3 $\mathrm{mM}$ lidocaine in more than $60 \%$ of atrial and ventricular cardiomyocytes isolated from F1759A-dTG mice (Figure 2, A, B, and D). This amount of residual lidocaine-resistant $\mathrm{Na}^{+}$current was not observed in NTG or single TG mice that were either rtTA-positive or F1759A-positive. The F1759A mutation prevented complete inactivation of $\mathrm{Na}_{\mathrm{v}} 1.5$, thereby increasing persistent $\mathrm{Na}^{+}$current in ventricular (Figure 2, A and E) and atrial (Figure 2, B and E) cardiomyocytes isolated from F1759A-dTG mice, as was previously reported when this mutation was studied in heterologously expressing HEK cells (17). Moreover, the voltage at which $\mathrm{Na}_{\mathrm{v}} 1.5$ is open $50 \%$ of the time $\left(\mathrm{V}_{50}\right)$ in F1759A-dTG ventricular cardiomyocytes was shifted in a hyperpolarizing direction by $3.3 \mathrm{mV}(P<0.05 ; n=23$ control; $n=13 \mathrm{dTG}$ ), and inactivation was shifted in the depolarizing direction by $1.9 \mathrm{mV}(P=0.07)$ leading to enhanced window current. The persistent $\mathrm{Na}^{+}$current was both resistant to ranolazine (Supplemental Figure 1A; supplemental material available online with this article; doi:10.1172/JCI84669DS1) and, after normalization to the amount of peak $\mathrm{Na}^{+}$current, correlated with the amount of lidocaine-resistant current in atrial and ventricular cells (Supplemental Figure 1, B and C), implying that the persistent $\mathrm{Na}^{+}$current was solely due to the TG F1759A-Na 1.5 channels and not due to posttranslational modifications of endogenous $\mathrm{Na}_{\mathrm{v}} 1.5$ channels.

The $\mathrm{Na}^{+}$current that persists throughout the AP plateau causes an increase of $\left[\mathrm{Na}^{+}\right]_{i}$, which can be exchanged for extracellular $\mathrm{Ca}^{2+}$ through the $\mathrm{Na}^{+}-\mathrm{Ca}^{2+}$ exchanger (NCX). We monitored intracellular $\mathrm{Ca}^{2+}$ cycling using Fura-2. Ventricular cardiomyocytes were paced at $1 \mathrm{~Hz}$. Imaging of $\mathrm{Ca}^{2+}$ transients of ventricular cardiomyocytes demonstrated that F1759A-dTG cardiomyocytes have significantly greater amplitude of $\mathrm{Ca}^{2+}$ transients than the control mice (Figure 2F).

Increased $\mathrm{Na}^{+}$influx is sufficient to cause atrial and ventricular cardiomyopathy. Elevated $\left[\mathrm{Na}^{+}\right]_{\mathrm{i}}$, caused by increased persistent $\mathrm{Na}^{+}$current, is a hallmark of both animal models and human heart failure (18-20). In humans, some gain-of-function SCN5A mutations are associated with familial dilated cardiomyopathy. It is not known, however, whether increased persistent $\mathrm{Na}^{+}$current is sufficient to cause a cardiomyopathy. We found that the atria and ventricles of F1759A-dTG mice progressively enlarged over several months after birth (Figure 3, A-E). We serially performed transthoracic echocardiograms of F1759A-dTG and littermate control mice. At 20-30 days of age, the left ventricular (LV) ejection fraction was reduced nonsignificantly by $14 \%$ compared with controls. Over the ensuing approximately 2 months, the LV ejection fraction (LVEF) modestly decreased, with a $27 \%$ and $35 \%$ relative reduction at $41-50$ days and 51-80 days, respectively (Figure $3 \mathrm{C}$ ). The reduc- 
A
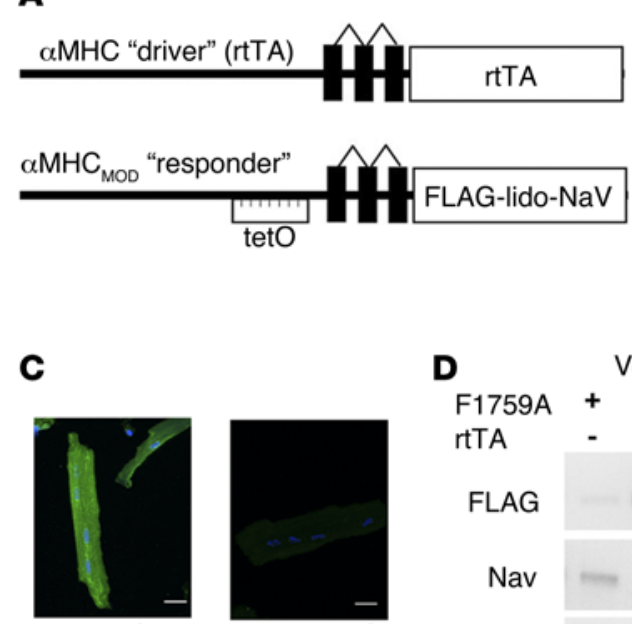

Anti-FLAG Ab No anti-FLAG Ab
D

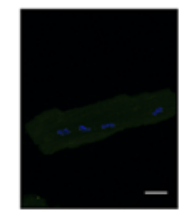

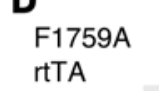

B

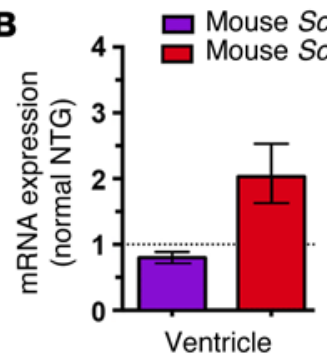

Mouse Scn5a

$5 a+$ human $S C N 5 A$

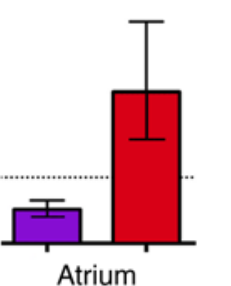

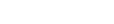

accelerates mitochondrial $\mathrm{Ca}^{2+}$ efflux and promotes reactive oxygen species (ROS) formation and oxidative stress $(22,23)$.

Spontaneous AF in F1759A$d T G$ mice. Extensive electrocardiographic analyses were performed to explore both the frequency and mechanisms of atrial and ventricular arrhythmias (Figure 4). A prolonged QT interval was observed in F1759A-dTG mice (Figure 4, A-D), consistent with the increased persistent $\mathrm{Na}^{+}$current leading to lengthening of the APD. The QT interval, in contrast, was normal in both single TG littermate genotypes (rtTA or F1759A) and NTG mice. A markedly increased frequency of premature ventricular complexes (PVCs) and nonsustained polymorphic VT was observed in F1759A-dTG mice by surface limb lead ECG and in nonanesthetized F1759A-dTG mice using tion in LVEF is likely multifactorial, probably due to both intrinsic contractility defects and secondarily due to a tachycardia-induced cardiomyopathy and/or the difficulty in assessing LV function in the setting of both $\mathrm{AF}$ and ventricular arrhythmias (see below). Atrial remodeling was detectable by echocardiography at very early stages after birth. The left atrial size was increased by $48 \%$ compared with controls at 20-30 days after birth, which persisted through at least 3 months of age (Figure 3D). Histopathological studies at 3-4 months of age demonstrated atrial hypertrophy; an increase of right and left atrial areas by $52 \%$ and $54 \%$, respectively (Figure 3, A and E); increased fibrosis (Figure 3F); and myocyte disarray (Figure 3, E and F) in the F1759A-dTG mice.

Transmission electron microscopy (TEM) images from mice at 6 and 12 weeks old also demonstrated atrial and ventricular structural remodeling in the F1759A-dTG mice (Figure 3G). At 6 weeks of age, mild disarray of the myofibrils was noted with increased collagen deposition in the atria, which progressed with glycogen deposition at 12 weeks of age. These changes mimic the findings observed in large animal models of AF and patients with AF. In the ventricle at both 6 and 12 weeks of age, the diameter of T-tubules (Figure $3 \mathrm{G}$, red arrows) was increased by 2.5-fold (Supplemental Figure 2), which has been observed in other animal models of heart failure (21). By 12 weeks of age, atrial and ventricular cardiomyocytes from the F1759A-dTG mice demonstrated mitochondrial injury, with circular and swollen mitochondria and ruptured outer membranes (Figure 3G). These findings establish that increased persistent $\mathrm{Na}^{+}$ current is sufficient to cause progressive structural changes in the atria and ventricles in vivo, including mitochondrial injury, poten-

telemetry (Figure 4G, upper tracing). Ventricular arrhythmias were never observed in the single TG or NTG mice.

In the 2 independent lines of F1759A-dTG mice, spontaneous $\mathrm{AF}$ - which was identified in anesthetized mice during a 3-minute recording period of a surface limb lead ECG (Figure $4 \mathrm{E})$ - was found as early as 5-6 weeks of age and by 10 weeks of age in greater than $80 \%$ of F1759A-dTG mice (Figure $4 \mathrm{~F}$ ). AF was present in many mice for the entire 3-minute recording period of a surface limb lead ECG (Figure 4E, upper tracing), but in others, the $\mathrm{AF}$ was paroxysmal (Figure $4 \mathrm{E}$, lower tracing). $\mathrm{AF}$ was never observed in NTG and single TG mice at any time point (Figure $4 \mathrm{~F})$. Since slowing of the heart rate under anesthesia could provoke atrial arrhythmias, especially due to the inverse relationship of heart rate and persistent $\mathrm{Na}^{+}$current, we implanted subcutaneous ECG telemeters. Spontaneous AF in nonanesthetized mice (Figure 4G, lower tracing) was detected in all 11 F1759A-dTG mice with implantable ECG telemeters, indicating a high penetrance. The average AF burden was $37 \% \pm 10 \%$ during a 20 -hour period (Figure $4 \mathrm{H}$ ) and the longest continuous episode of AF was 1 hour, 52 minutes. Spontaneous AF was never observed in NTG or single TG littermates implanted with ECG telemeters $(n=10)$. These findings are in marked contrast to the vast majority of previously reported mouse models of $\mathrm{AF}$, including the $\triangle \mathrm{KPQ} \mathrm{Na}{ }^{+}$channel knock-in mice, in which atrial arrhythmias could only be elicited by very aggressive pacing and were not sustained for longer than several seconds (24-26). Our results imply that increased persistent $\mathrm{Na}^{+}$current is sufficient to alter the substrate and serve as a triggering factor for the initiation and perpetuation of $\mathrm{AF}$. 
A

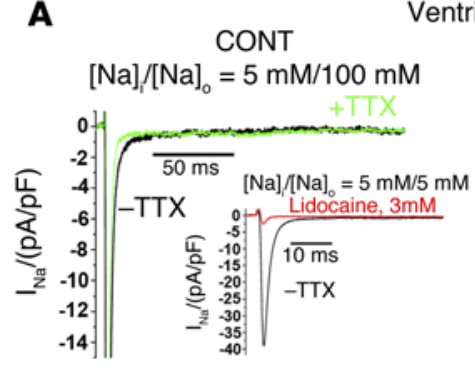

F1759A dTG

$[\mathrm{Na}]_{i} /[\mathrm{Na}]_{0}=5 \mathrm{mM} / 100 \mathrm{mM}$
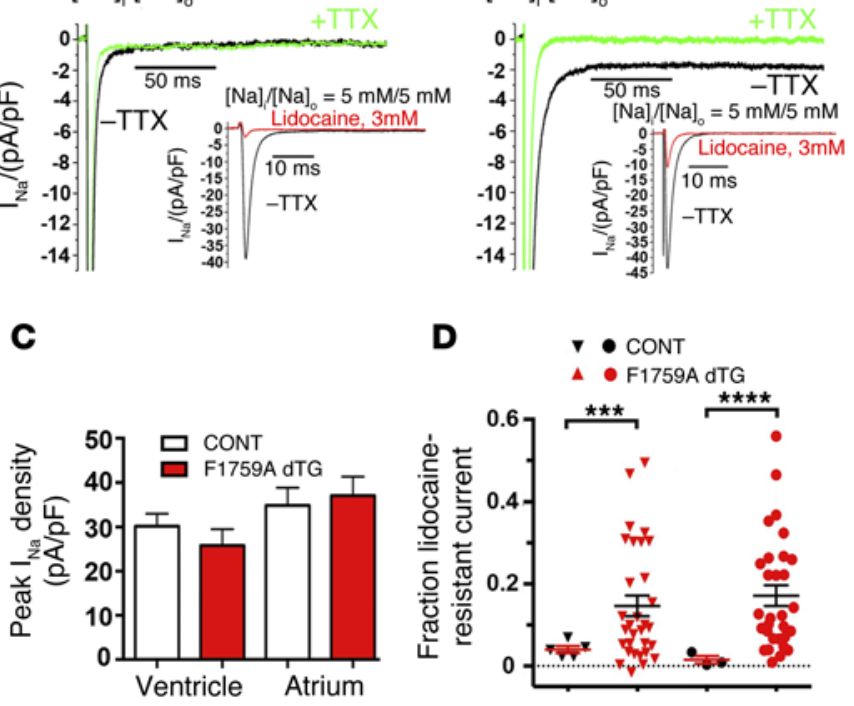

D

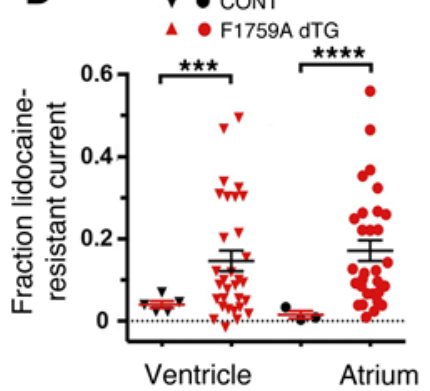

B

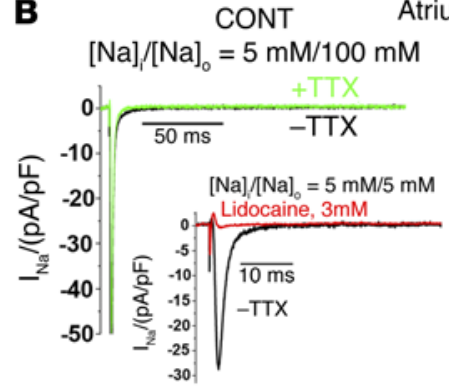

F1759A dTG

$[\mathrm{Na}] /[\mathrm{Na}]_{0}=5 \mathrm{mM} / 100 \mathrm{mM}$
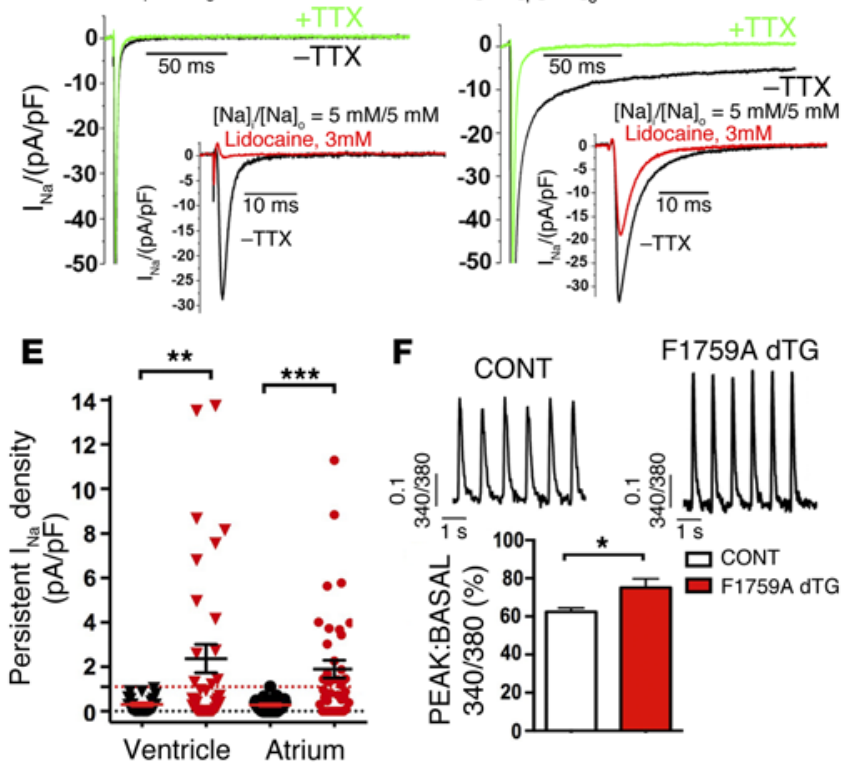

Figure 2. F1759A- $\mathrm{Na}_{\mathrm{v}} 1.5$ increases persistent $\mathrm{Na}^{+}$current in atria and ventricles. (A and B) Exemplar whole cell $\mathrm{Na}^{+}$current $\left(\mathrm{I}_{\mathrm{Na}}\right)$ traces of ventricular $(\mathbf{A})$ and atrial (B) cardiomyocytes isolated from control (CONT, single TC and NTG) and F1759A-dTC mice. Persistent $I_{\mathrm{Na}}$ was evaluated with a 190-ms depolarization from a holding potential of -110 to $-30 \mathrm{mV}$ in the absence (black) and presence (green) of $20 \mu \mathrm{M} \mathrm{TTX;} 5 \mathrm{mM} \mathrm{Na}^{+}$was used in the intracellular solution, and $100 \mathrm{mM} \mathrm{Na}$ was used in the extracellular solution. Insets: For the assessment of peak $\mathrm{I}_{\mathrm{Na}}$ and the fraction of lidocaine-resistant current, whole cell current traces were recorded with $5 \mathrm{mM} \mathrm{Na}^{+}$in both extracellular and intracellular solutions, in the absence (black) and presence (red) of 3 $\mathrm{mM}$ lidocaine. (C) Bar graph of peak $\mathrm{I}_{\mathrm{Na}}$ density recorded with $5 \mathrm{mM}$ external $\mathrm{Na}^{+}$. Data are presented as mean $\pm \mathrm{SEM}$. (D) Graph of fraction of peak $\mathrm{I}_{\mathrm{Na}}$ resistant to $3 \mathrm{mM}$ lidocaine. ${ }^{* *} P<0.001,{ }^{* * *} P<0.0001 ; t$ test. (E) Graph of persistent $\mathrm{I}_{\mathrm{Na}}$. Red dashed line is maximal persistent $\mathrm{I}_{\mathrm{Na}}$ in cardiomyocytes isolated from CONT mice. Data are presented as mean $\pm \mathrm{SEM}$. ${ }^{* *} P<0.01,{ }^{* * *} P<0.001 ; t$ test. (F) Representative traces of Ca ${ }^{2+}$ transients. Bar graph of $\mathrm{Ca}^{2+}$ transients of littermate control (CONT) and F1759A-dTC mice. Data are presented as mean \pm SEM. ${ }^{*} P<0.05 ; t$ test. $n=34$, single TC and NTG ventricular cardiomyocytes; $n=107$, F1759A-dTC cardiomyocytes.

Surface optical voltage mapping of AF. To elucidate the electrophysiological mechanisms responsible for $\mathrm{AF}$, we performed epicardial surface optical voltage mapping of the anterior surface of 12 explanted Langendorff-perfused hearts of F1759AdTG mice and 10 hearts of single TG and NTG littermate control hearts. Prior to euthanasia, AF was confirmed by surface limb lead ECG in all F1759A-dTG mice. Spontaneous AF, sustained for the duration of the ex vivo experiments in $75 \%$ and paroxysmal in $25 \%$, was demonstrated using a pseudo-ECG in all explanted Langendorff-perfused F1759A-dTG hearts. In contrast to most small and large animal studies, pacing, drugs, or alterations of the electrolyte composition of the perfusion solution were not required for induction or perpetuation of $\mathrm{AF}$ ex vivo. Spontaneous $\mathrm{AF}$ was never detected in any of the 10 explanted littermate control hearts. In the paroxysmal AF group, spontaneous AF was rarely interrupted by brief bursts of sinus rhythm, enabling us to compare the APD of F1759A-dTG mice to littermate controls. The $\mathrm{APD}_{80}$ of the F1759A-dTG mice was prolonged 4-fold in the right atrium and 2.5-fold in the left atrium (Figure 5, A and B). Increased heterogeneity of the APD was apparent in both the right and left atria (Figure 5, C and D), likely caused by the variable expression of the F1759 $\mathrm{A} \mathrm{Na}^{+}$channels in atrial cardiomyocytes, as observed in the cellular electrophysiological studies (Figure 2). Conduction velocity was not different in the NTG and F1759A-dTG mice (Supplemental Figure 3).
There is growing interest in the substrates that sustain $\mathrm{AF}$ after it has been triggered. Despite years of studies, there is still no agreement as to the fundamental mechanisms that sustain $\mathrm{AF}$. One theory is that the electrophysiological substrate for AF in many patients includes rotational circuits or focal triggers. Rotors are spiral waves with patterns of circular reentry for one or more cycles around a phase singularity (27). The core occupies regions of myocardium that are capable of sustaining the highest possible frequency of reentrant activity. The spiral waves, radiating at high velocity, generate disorganized activity caused by conduction block between the regions encompassed by the principal rotor and regions with longer refractory periods. We hypothesized that the regional heterogeneity in APD would serve as substrate to enable rotor formation and generate the secondary disorganized activity.

We utilized phase mapping to quantify rotor dynamics including phase singularity points. During both sustained and paroxysmal AF ex vivo, single and, at times, multiple simultaneous rotors of (+) or (-) chirality with rates up to approximately $53 \mathrm{~Hz}$ were recorded in the right atria (Figure 5, E-G, and Supplemental Video 1) and left atria (Figure 5, H-J, and Supplemental Video 2). Rotors were identified if the phase singularity persisted for long enough for the phase pattern to cycle at least 2 times around the phase singularity. We also observed, in one heart, a concurrent rotor in the right atrium and a focal repetitive wave in the left atrium, which may represent either focal-triggered activity or a spiral wave/rotor in which the 
A

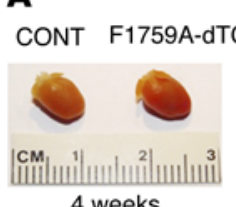

CONT F1759A-dTG

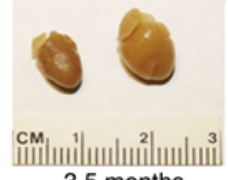

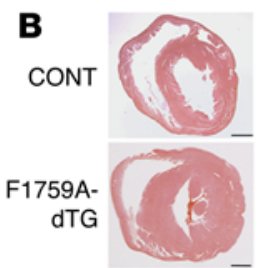

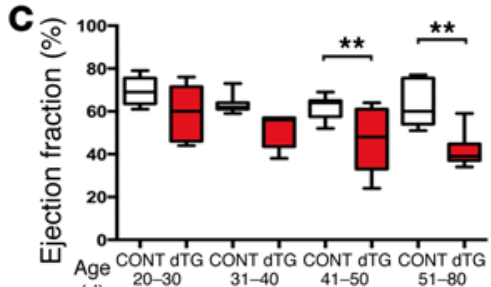

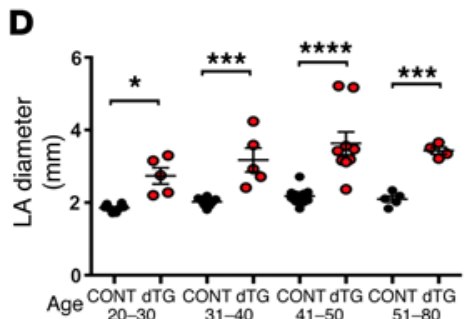

(d)
E CONT

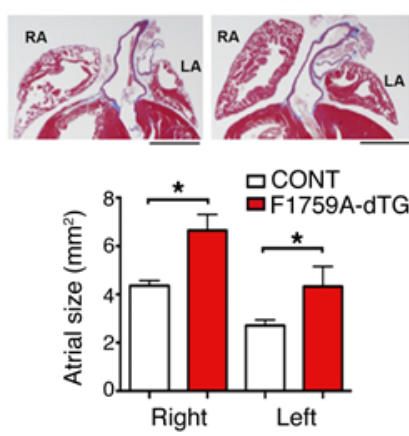

F

\section{F}
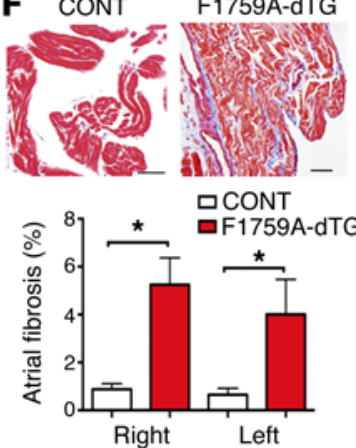

G

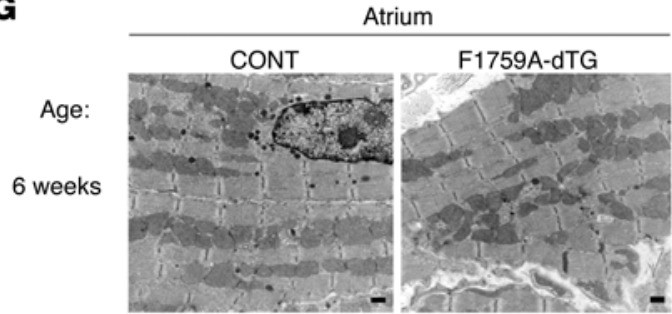

12 weeks
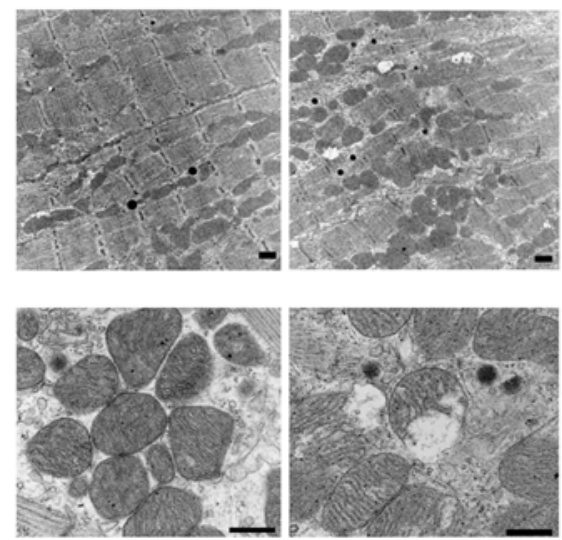

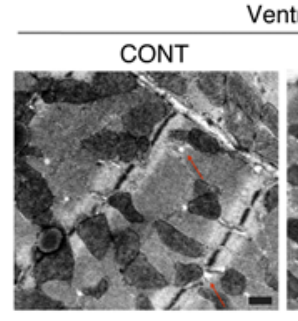

Ventricle
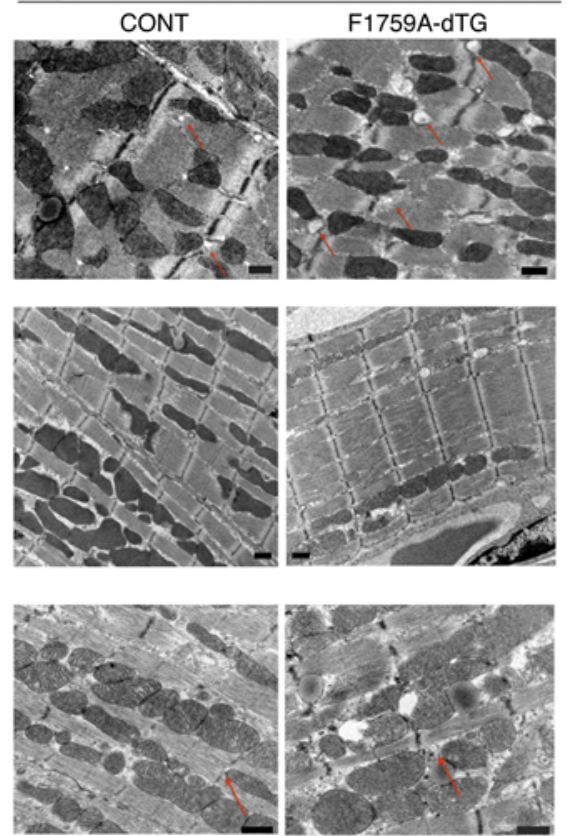

Figure 3. Increased persistent $\mathrm{Na}^{+}$current is sufficient to initiate structural changes in the atria and ventricles.

(A) Photographs of littermate control (CONT) and F1759A-dTC hearts at 4 weeks and 3.5 months after birth. (B) $\mathrm{H} \& \mathrm{E}$-stained cross section of ventricle. Scale bars: $1 \mathrm{~mm}$. (C and $\mathbf{D}$ ) Graphs of ejection fraction and LAD (in $\mathrm{mm}$ ) of littermate control and F1759A-dTC mice, assessed by echocardiography at ages shown. Data are presented as mean \pm SEM. $n \geq 5$ mice per age group per genotype. ${ }^{*} P<0.05,{ }^{*} P<0.01$, ${ }^{* * *} P<0.001$, ${ }^{* *} P<0.0001$. (E) H\&E stain of right and left atria showing bi-atrial enlargement in F1759A-dTC mice. Scale bars: $1 \mathrm{~mm}$. Bar graph of left atrial and right atrial area of littermate control and F1759A-dTC mice at 3-4 months of age. Data are presented as mean \pm SEM. ${ }^{*} P<0.05$; $t$ test. $n=5$ for each group. (F) Masson's trichrome stain of atria of littermate control and F1759A-dTC mice. Scale bars: $50 \mu \mathrm{m}$. Bar graph quantifying atrial fibrosis. Data are presented as mean \pm SEM. ${ }^{*} P<0.05$; $t$ test. $n \geq 5$ for each group. (C) Representative 2-dimensional TEM images ( $n=2$ for dTC and littermate control) showing left to right, gross morphology of atrial and ventricular samples at 6 weeks (first row) and 12 weeks (second and third rows) in littermate control mice compared with dTC mice. At 6 weeks, dTC mice show signs of myofibril disarray with loss of congruous parallel myofibrils in the atrium. By 12 weeks of age, atrial and ventricular cardiomyocytes from the F1759A-dTC mice demonstrated mitochondrial injury, with circular and swollen mitochondria and ruptured outer membranes. In the ventricle, the red arrows point to T-tubule cross sections, which are larger in the dTC mice compared with littermate control. Scale bars: $500 \mathrm{~nm}$. phase singularity was not imaged, possibly because of the limits of surface epicardial mapping (Supplemental Video 3). In the hearts of sustained and paroxysmal AF, the rotors often showed meandering (Supplemental Figure 4 and Supplemental Videos 1-3), which has clinical implications for therapies since an ablative strategy with a wide area that encloses the area of migration of the rotor may have better success than a more limited focus. The area around the rotating axes of the wave front exhibited progressive phase changes (Figure 5, F and I), and a region close to the core of the rotor, electrogram 6, displayed bidirectionality, consistent with a spiral wave.

To determine the dominant frequency (DF), spectral and fractionation analyses of optical and electric signals were performed on their power spectra by fast Fourier transformation. The area of highest DF colocalized with the region of the rotor. Rotors in the right atrium had a DF of $10-53 \mathrm{~Hz}(n=8)$ and rotors in the left atrium had a DF of $23-40 \mathrm{~Hz}(n=7)$. When rotors were simultaneously present in the right and left atria, they had different DF, implying that they were independent. The regularity index (RI), defined as the repetitiveness over time of the morphology of the detected activation waves (28), was used to quantify rotor cyclicity. The RI strongly correlated with DF (Supplemental Figure 5), implying that the most stable rotors were also the most regular rotors, which has been observed in patients with persistent $\mathrm{AF}$ (29). In $20 \%$ of F1759A-dTG hearts with AF, however, rotational circuits were not observed, although the optical mapping was limited to a single surface.

NCX inhibition reduces frequency of atrial and ventricular arrhythmias. The relatively low efficacy of pharmaceuticals and 
A Surface ECG control

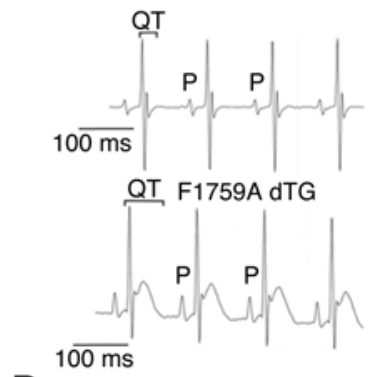

B

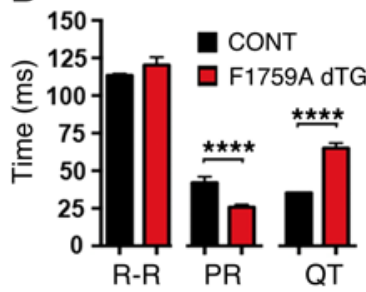

C Telemetry ECG control

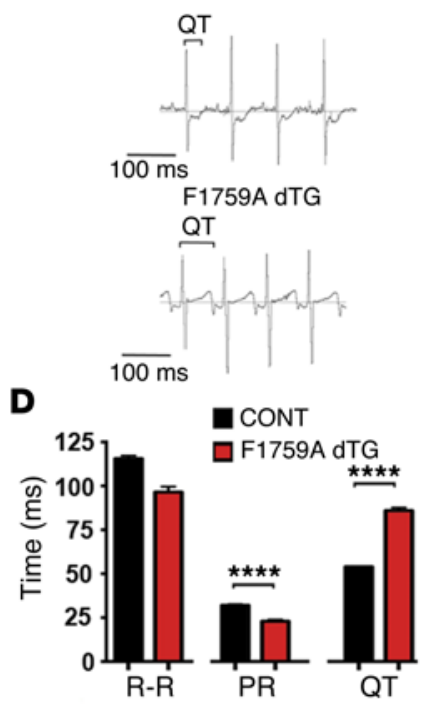

1759A dTG

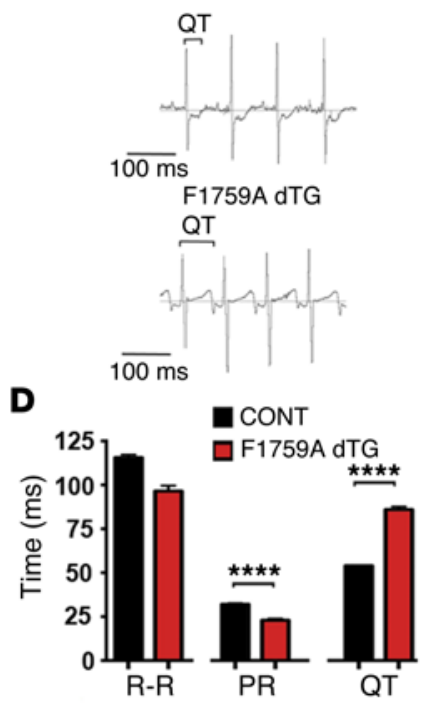

$\mathbf{F}$
$\mathbf{E}$
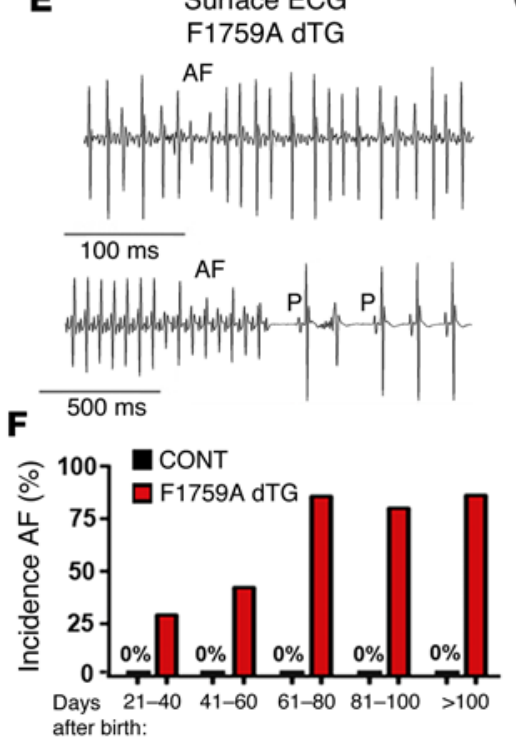

G Telemetry ECG

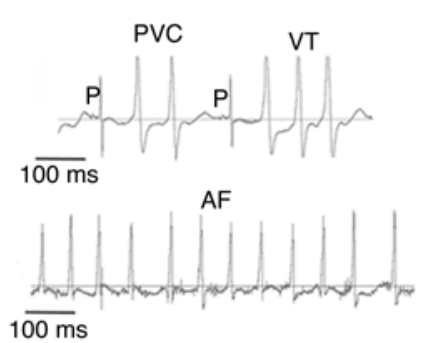

H

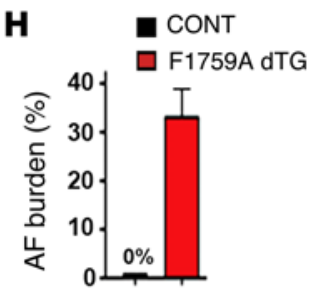

Figure 4. Prolonged QT interval and spontaneous AF in F1759A-dTG mice. (A) Representative limb-lead surface electrocardiograms of isofluraneanesthetized littermate control and F1759A-dTC mice. The QT interval is marked by brackets. (B) Bar graph of R-R, PR, and QT intervals from isoflurane-anesthetized littermate control mouse and F1759A-dTG mouse. Data are presented as mean \pm SEM. ${ }^{* * * *} P<0.0001 ; t$ test. (C) Representative telemetry recordings of nonanesthetized littermate control and F1759A-dTG mice. (D) Graph comparing R-R, PR, and QT intervals of nonanesthetized littermate control and F1759A-dTC mice from telemetric ECG recordings. ${ }^{* * *} P<0.0001$; $t$ test. (E) Representative surface electrograms of isoflurane-anesthetized F1759A-dTC mice showing AF. In the lower trace, paroxysmal AF was recorded, with P waves at the right side of the recording. (F) Bar graph showing incidence of AF at range of days after birth. Surface electrograms of isoflurane-anesthetized mice were recorded for 3 minutes. AF was considered present if the duration of AF persisted for $>10$ ventricular complexes. CONT ( $\operatorname{lng}$ le TG + NTC): $n=27,2-40$ days; $n=8,41-60$ days; $n=5$, 61-80 days. F1759A-dTG: $n=26,21-40$ days; $n=23,41-60$ days; $n=17,61-80$ days; $n=18,81-100 ; n=9$, $>100$ days. Each age group consisted of a distinct set of mice. (G) Representative telemetry recordings of nonanesthetized F1759A-dTG mice showing VT (upper tracing) and AF (lower tracing). (H) Graph showing percent of AF during 20-hour recording in control and F1759A-dTC mice. $n=3$ mice, CONT; $n=8$, F1759A-dTC (5 were male and 3 were female).

radiofrequency ablation/surgery and high rates of recurrence have plagued clinical treatment of AF. The F1759A-dTG mice have the potential to offer a means to test novel therapeutic approaches, either using pharmacological tools or genetically altered mice. The mechanism for the increased persistent $\mathrm{Na}^{+}$current in $\mathrm{AF}$ patients (11) is not known. It may be due to increased $\mathrm{Na}_{\mathrm{v}} 1.1$ expression or due to increased CaMKII phosphorylation or oxidative stress, both of which are known to increase persistent $\mathrm{Na}^{+}$ current (30-33). A large clinical trial (MERLIN-TIMI 36) showed that ranolazine, an inhibitor of persistent current, may reduce the frequency of paroxysmal AF in patients with acute coronary syndrome (34). F1759A- $\mathrm{Na}^{+}$channels are resistant to therapeutic blood levels of ranolazine (Supplemental Figure 1A).

Prior studies have demonstrated that a relatively specific inhibitor of the NCX, SEA-0400, suppressed dofetilide-induced torsade de pointes in anesthetized dogs (35) and in Langendorffperfused rabbit hearts with drug- and hypokalemia-induced models of LQT2 and LQT3 (36), possibly by reducing $\left[\mathrm{Ca}^{2+}\right]_{\mathrm{i}}$ (Figure 6A). The efficacy of NCX inhibitors in AF, however, is unknown. We determined the fraction of time in $\mathrm{AF}$ vs. sinus rhythm during a 20-hour period before and after a single $0.4 \mathrm{mg} / \mathrm{kg}$ i.p. injection of SEA-0400 by manually scanning the entire ECG record. We found that SEA-0400 markedly reduced the fraction of AF over the 20 -hour period in 4 of 5 mice (Figure $6, \mathrm{~B}-\mathrm{D}$ ). The mean reduction in AF burden during the 20-hour recording period for the 4 responding mice was $69 \%$ and was $59 \%$ for all 5 mice (Figure 6D).
The single i.p. injection of SEA-0400 also markedly reduced the frequency of PVCs by 76\% (Figure 6E), without affecting the QT interval (Figure 6F). Thus, a single injection of SEA0400 markedly reduced $\mathrm{AF}$ and ventricular ectopy in vivo without lengthening the QT interval, which frequently limits administration of many antiarrhythmic drugs, including ranolazine.

\section{Discussion}

Our data show that gain-of-function $\mathrm{Na}^{+}$channel perturbations are sufficient to cause structural and functional changes within the atrium and ventricles of mice. This is consistent with emerging observations that persistent $\mathrm{Na}^{+}$current plays an important role in progression of heart failure (31). Both gain-of-function and loss-offunction $\mathrm{Na}^{+}$channel dysfunctions are associated with familial dilated cardiomyopathy, and persistent $\mathrm{Na}^{+}$current is associated with acquired diastolic and systolic dysfunction and electrical instability. For instance, an R222Q SCN5A gain-of-function variant, which left-shifted the steady state parameters of activation and inactivation, caused reversible ventricular ectopy and a dilated cardiomyopathy, which were substantially reversed with amiodarone or flecainide (37). The F1759A-dTG mice mimic many of these structural and functional abnormalities, including cardiac enlargement and ventricular dysfunction, and our studies reveal, at least in part, the downstream mechanisms by which gain-of-function SCN5A mutants cause a dilated cardiomyopathy and substrates for both atrial and ventricular arrhythmogenesis. Inhibiting reverse 
A

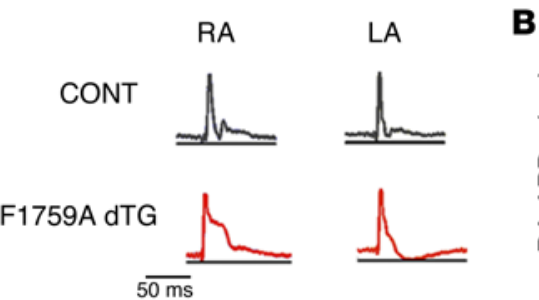

C
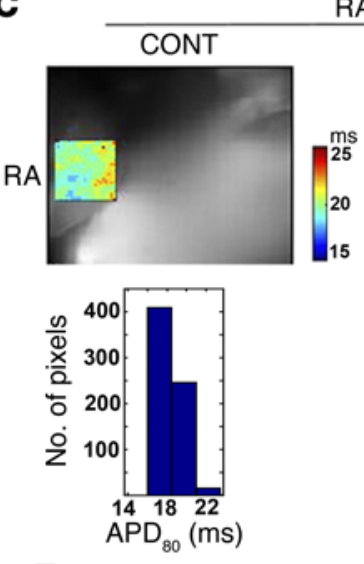

E

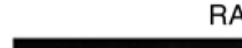

F1759A dTG
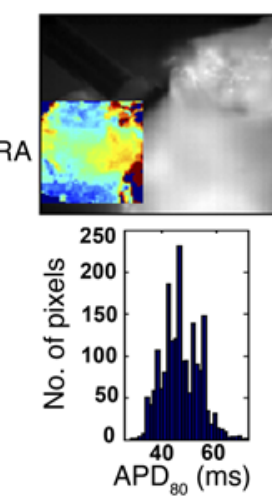

RA

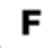

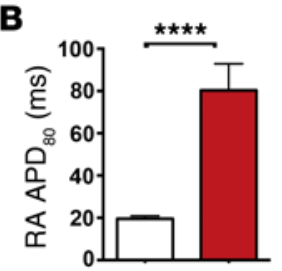

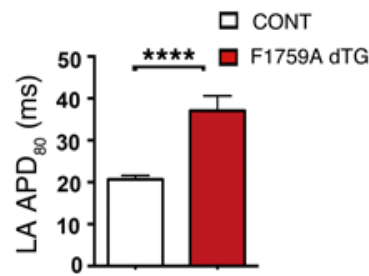

D

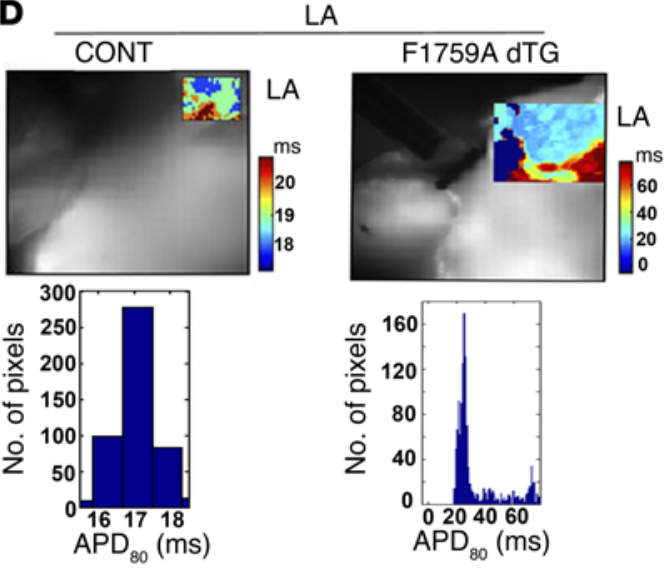

G

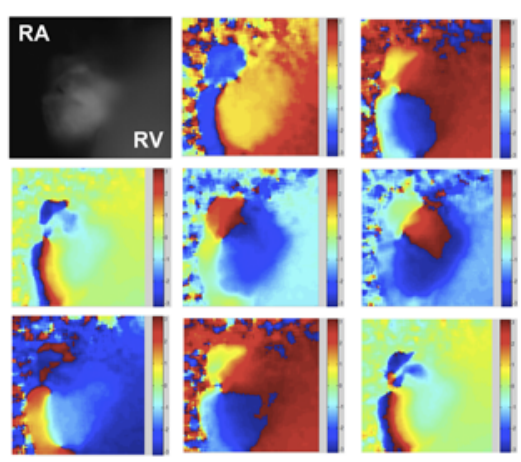

H

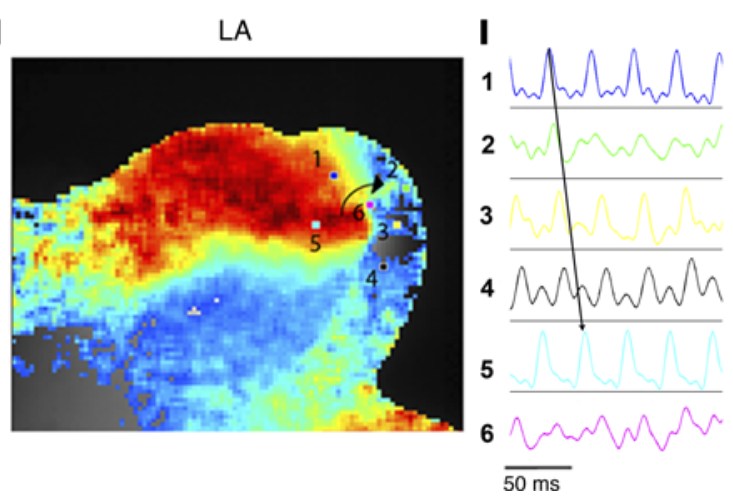

J
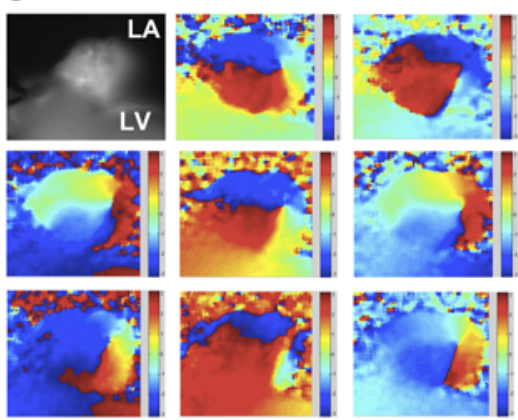

Figure 5. Optically recorded atrial APs and voltage maps of AF. (A and B) Optically recorded APs from right atrium (RA) and left atrium (LA) of control and F1759A-dTC mice in sinus rhythm (A). Bar graphs showing right and left atrial APD ${ }_{80}$ of control mice $(n=10$ mice) and F1759A-dTC mice $(n=3$ mice) (B). Data are presented as mean \pm SEM. ${ }^{* * *} P<0.0001 ; t$ test. The mean atrial rate in sinus rhythm: NTC mice, $338 \pm 9.5$ beats per minute; F1759A-dTC mice, $314 \pm 9.8$ beats per minute. (C and D) Representative APD regional maps and all point histograms of control (CONT) and F1759A-dTC mice showing increased $A P D_{80}$ dispersion in the RA and LA of F1759A-dTC mice. Color legend of $A P D_{80}$ (ms) is shown to the right of each optical map. (E-J) Optical voltage map of AF in Langendorff-perfused F1759A-dTC explanted heart. (E and $\mathbf{H}$ ) Phase maps of concurrent but independent rotors in the RA and LA. (F and I) Optical APs (electrograms 1-5) around the rotor demonstrate progressive circular reentry around the phase singularity (electrogram 6). (G and J) Progressive phase map of the RA (G) and LA (J) every $10 \mathrm{~ms}$ of the phase movie. Red, depolarization; blue, repolarization. 

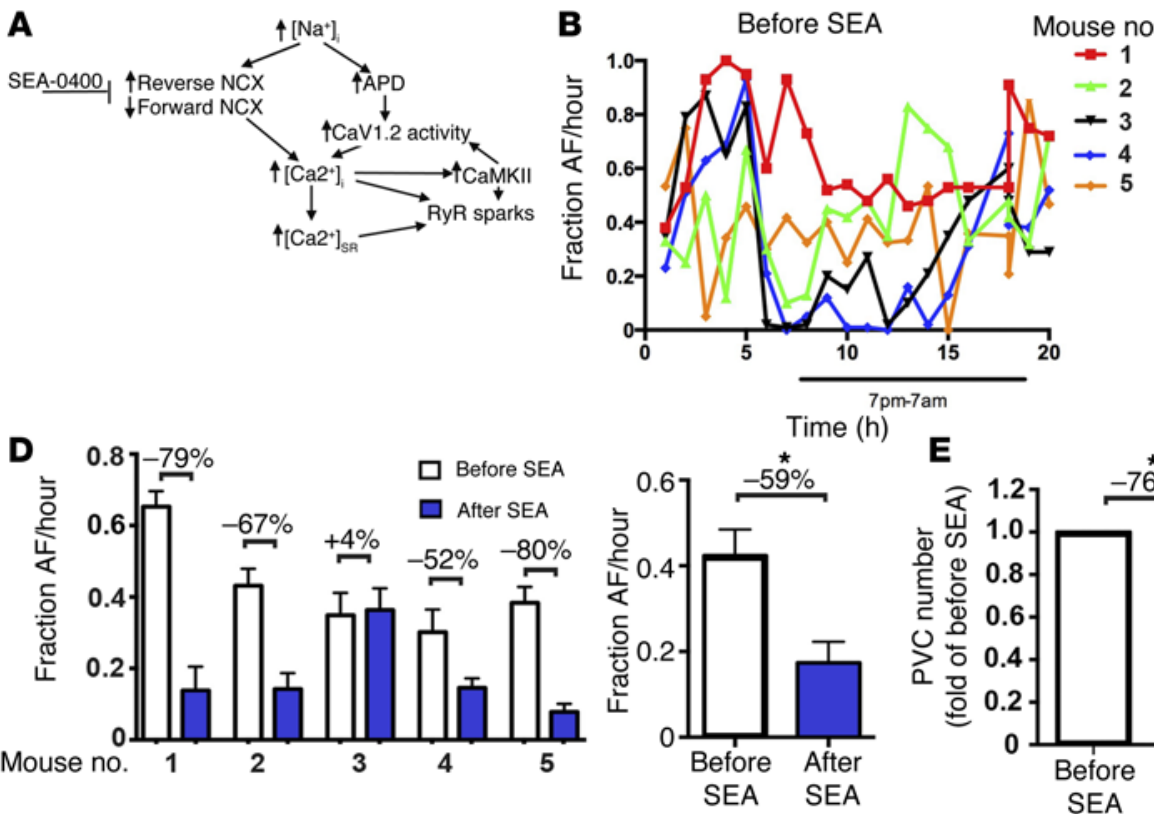

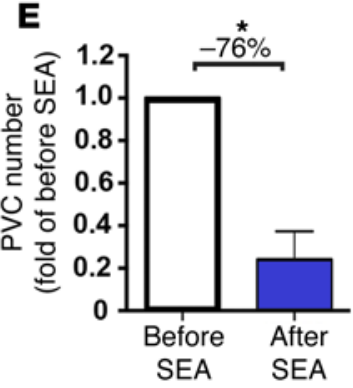

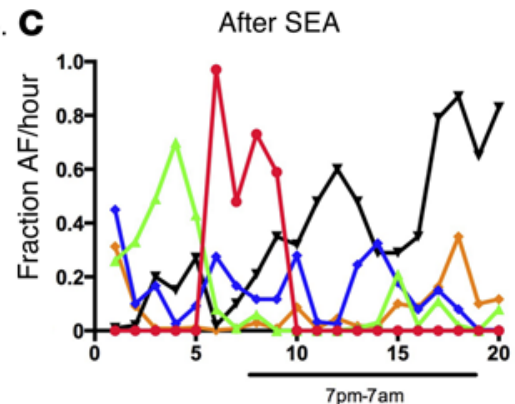

$\mathbf{F}$

Time (h)

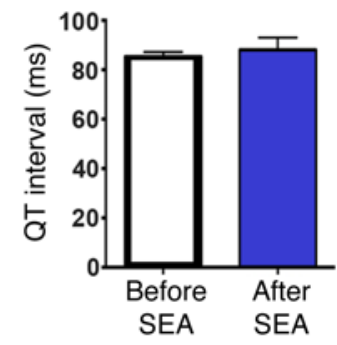

Figure 6. Inhibition of NCX attenuates atrial and ventricular arrhythmogenesis in F1759A-dTC mice. (A) Schematic depicting proposed mechanisms of arrhythmogenesis in F1759A-dTC mice. (B) Diary plot of fraction of AF/hour during a 20-hour period for 5 male F1759A-dTC mice. Burden of AF was determined by manually reviewing the entire 20-hour period. (C) Diary plot of fraction of AF/hour for same 5 male F1759A-dTC mice during a 20-hour period after i.p. injection of SEA-0400. (D) Graph (left) summarizing fraction of AF during 20-hour period before (white bars) and after (blue bars) single i.p. injection of SEA-0400 for each mouse. Percent change is shown above each data pair. Graph (right) showing fraction AF/hour before and after SEA injection for the 5 F1759A-dTC mice. Data are presented as mean $\pm S E M .{ }^{*} P<0.05 ; t$ test. (E) Quantification of number of PVC normalized to pre-SEA. Data are presented as mean $\pm \mathrm{SEM}$. ${ }^{*} P<0.05$; $t$ test. $(\mathbf{F})$ Bar graph showing $\mathrm{QT}$ interval before and after SEA- 0400 .

mode $\mathrm{Na}^{+}-\mathrm{Ca}^{2+}$ exchange acutely reduced the burden of both spontaneous atrial and ventricular arrhythmias, identifying a potentially new pharmacological approach for treatment of AF in humans.

It is likely that the primary effects of incomplete $\mathrm{Na}_{\mathrm{v}} 1.5$ inactivation on cardiomyocyte electrophysiology, namely prolongation and dispersion of the APD, and the secondary downstream effects on chamber enlargement, fibrosis, and mitochondrial injury/ROS synergistically cause the unique phenotype of spontaneous and prolonged episodes of AF in mice, mimicking human disease. In the vast majority of previously reported mouse models of AF, atrial arrhythmias could only be elicited by very aggressive burst pacing, suggesting that while there may be a substrate for atrial arrhythmia, this can be well tolerated and undetected in the absence of a triggering factor (25). AF is defined in these studies by a duration of at least 1 second, and most previously reported mouse models demonstrated these relatively short episodes of AF (25). Conversely, the presence of a triggering factor but absence of structural changes also preclude the perpetuation of sustained atrial arrhythmia. Uniquely, the F1759AdTG mice show spontaneous and relatively prolonged episodes of $\mathrm{AF}$ in vivo and ex vivo, implying that both the substrate and triggering factors induced by the dysfunctional $\mathrm{Na}^{+}$channels are intrinsic to the heart. Moreover, the electrophysiological mechanisms - namely rotors, waves, and wavelets - were frequently observed in isolated hearts from these mice, similar to the findings in patients with $\mathrm{AF}$ and the classic large animal models of AF (38-40). Tissue heterogeneities in excitability, repolarization, conduction velocity, or even dynamic properties such as AP alternans can cause unidirectional conduction block, which can be the basis for initiating and perpetuating rotors (27).

The presence of spontaneous and prolonged episodes of atrial arrhythmias in this mouse model differs from prior studies using other mouse models of defective $\mathrm{Na}^{+}$channel inactivation. For instance, the $\triangle \mathrm{KPQ}$ heterozygous knock-in mice were not reported to have spontaneous or prolonged episodes of $\operatorname{AF}(41,42)$, and the frequency of extrastimuli-induced atrial arrhythmias was not different compared with control mice, although a very aggressive protocol of repetitive, intermittent high-rate burst stimulation resulting in short-long-short sequences provoked atrial tachyarrhythmias in approximately two-thirds of $\triangle \mathrm{KPQ}$ mice (43). Left atrial diameter (LAD) was mildly increased by $10 \%$ in $\triangle \mathrm{KPQ}$ mice $>5$ months old. In other studies, TG mice lines expressing the $\mathrm{WT} \mathrm{Na}_{\mathrm{v}} 1.5$ or the LQT3-mutant N1325S did not develop atrial arrhythmias $(44,45)$.

Patients with lone AF and permanent AF have increased persistent $\mathrm{Na}^{+}$current, and patients with LQT3 syndrome have an increased incidence of AF (9-11), demonstrating the relevance of this animal model to humans. Although mice have a different cardiac ion channel profile compared with humans, with a markedly higher basal heart rate, the importance of $\mathrm{Na}_{\mathrm{v}} 1.5$ and its complete inactivation is conserved between species. The F1759A-dTG mice demonstrate the structural and electrophysiological changes that are known to be associated with AF in humans and are thus a unique model to explore the molecular mechanisms responsible for the most common arrhythmia in humans. Taken together, the enhanced $\mathrm{Na}^{+}$influx, via incomplete $\mathrm{Na}_{\mathrm{v}} 1.5$ inactivation, and APD prolongation with dispersion; the resultant downstream maladap- 
tive effects including increased intracellular $\left[\mathrm{Ca}^{2+}\right]$, via increased $\mathrm{Ca}^{2+}$ entry and reverse mode $\mathrm{Na}^{+}-\mathrm{Ca}^{2+}$ exchange; and the structural changes within the atrium are likely responsible and sufficient for uniquely triggering and perpetuating AF.

\section{Methods}

General experimental approaches. All experimental procedures and analyses were performed in a blinded fashion. No data points, samples, or mice were excluded from the study.

Mouse model. The F1759A line was generated by fusing human heart sodium channel $\alpha$-subunit cDNA (hH1) $(46,47)$ to clone 26 vectors containing the modified murine $\alpha-\mathrm{MHC}$, tetracycline-inducible promoter (responder line) $(14,48)$. SCN5A was engineered to be lidocaine resistant by introducing an Ala-substitution at position F1759, and a 3X FLAG epitope was ligated in frame to the $\mathrm{N}$-terminus. The F1759A TG-positive mice in a B6CBA/F2 hybrid background were bred with cardiac-specific rtTA mice in a FVB/N background (obtained via Mutant Mouse Regional Resource Center) (15) to generate F1759A-dTG mice and single TG (rtTA-positive or F1759A-positive) littermate controls.

Telemetry and ECG analysis. Telemetry devices (model ETA-F10; Data Sciences International) were implanted in 4- to 11-month-old mice. Recordings were started 1 week after implantation. PR, RR, QRS, and QT intervals were measured manually using Ponemah 3 software (Data Sciences International). Three blinded, independent reviewers assessed the burden of atrial and ventricular arrhythmias. Subcutaneous 4-lead electrocardiograms of isoflurane-anesthetized mice were performed using EMKA ECG and recorded using Iox. AF was defined as absence of $\mathrm{P}$ waves and irregular R-R intervals for $>1$ second. SEA-0400 (ChemScene), dissolved in DMSO, was administered via i.p. injection.

Quantitative PCR ( $q$ PCR). PCR was performed using Applied Biosystems StepOne Plus RT-PCR system and inventoried following inventoried primers/mouse actin B, Mm01205647_g1; mouse

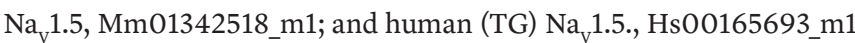
(Applied Biosystems). A custom set of primers was created by Applied Biosystems to detect the combined expression of mouse Scn5a and human SCN5A. PCR was performed, in duplicate, for 40 cycles with automated detection of crossing threshold. Results were presented as fold-difference for each gene against actin using $2^{-\Delta \Delta C T}$ and then normalized to NTG mice.

Immunoblots and immunofluorescence. Cardiomyocytes were homogenized in a $1 \%$ Triton X-100 buffer containing (in $\mathrm{mM}$ ): 50 Tris-HCl (pH7.4), $150 \mathrm{NaCl}, 10$ EDTA, 10 EGTA, and protease inhibitors. The lysates were incubated on ice for 30 minutes and centrifuged at $20,800 \mathrm{~g}$ at $4^{\circ} \mathrm{C}$ for 10 minutes. Supernatants were collected. Proteins were size-fractionated on SDS-PAGE, transferred to nitrocellulose membranes, and probed with anti-FLAG (catalog A8592, SigmaAldrich), anti-Na 1.5 (catalog ASC-005, Alomone) and anti-tubulin (catalog sc-12462-R, Santa Cruz Biotechnology Inc.) antibodies. Detection and quantification was performed with a CCD camera (Carestream) and ImageQuant software (GE Healthcare), respectively. For immunofluorescence, isolated cardiomyocytes were fixed for 15 minutes in $4 \%$ paraformaldehyde. Indirect immunofluorescence was performed using a 1:200 rabbit anti-FLAG antibody (Sigma-Aldrich) and 1:200 FITClabeled goat anti-rabbit antibody (Sigma-Aldrich). Images were acquired using a confocal microscope.

Cellular electrophysiology. Cardiomyocytes were isolated as described previously (49) from mice at least 8 weeks of age. Prior to euthanasia, surface ECG was obtained documenting AF in all F1759A-dTG mice. Experiments were performed at room temperature. Membrane currents from noncontracting rod-shaped cells with clear striations were measured by the whole-cell patch-clamp method using a MultiClamp 700B amplifier (Axon Instruments). The pipette resistance was 0.4-1.0 $\mathrm{M} \Omega$ in order to minimize voltage clamp error. The liquid junction potential was corrected, and series resistance was compensated up to $60 \%$. The leak current was subtracted using a $\mathrm{P} / 4$ protocol. The intracellular (pipette) solution contained (in mM): $5 \mathrm{NaCl}, 15 \mathrm{CsCl}, 115 \mathrm{CsF}, 10$ HEPES, and 10 BAPTA (pH 7.4) titrated with $\mathrm{CsOH}$. For persistent $\mathrm{Na}^{+}$current determinations, the bath solution contained (in $\mathrm{mM}$ ): $100 \mathrm{NaCl}, 45 \mathrm{TEA}-\mathrm{Cl}$, 10 HEPES, $1 \mathrm{MgCl}_{2}, 1 \mathrm{CaCl}_{2}$, and 5 glucose ( $\mathrm{pH}$ 7.4) titrated with $\mathrm{CsOH}$. The bath solution was then changed to reduce $\mathrm{Na}^{+}$concentration to minimize $\mathrm{Na}^{+}$current. It contained (in $\mathrm{mM}$ ): $5 \mathrm{NaCl}, 140$ TEA-Cl, 10 HEPES, $1 \mathrm{MgCl}_{2}, 1 \mathrm{CaCl}_{2}$, and 5 glucose ( $\mathrm{pH}$ 7.4) titrated with $\mathrm{CsOH}$. Lidocaine (3 $\mathrm{mM})$ was superfused to determine the lidocaine-resistant current. Persistent current was evaluated by $190-\mathrm{ms}$ depolarization from -100 to $-30 \mathrm{mV}$ in the absence and presence of $20 \mu \mathrm{M}$ tetrodotoxin (TTX, Tocris Bioscience) (50). Digital subtraction of the time-averaged responses in the absence and presence of TTX yielded a small TTX-sensitive persistent $\mathrm{Na}^{+}$current. The mean value of the last $10 \mathrm{~ms}$ of the 190 -ms pulse was normalized to the peak $\mathrm{Na}^{+}$current recorded using $5 \mathrm{mM} \mathrm{Na}^{+}$in both intracellular and extracellular solutions.

$\mathrm{Ca}^{2+}$ transients. Myocytes were loaded with Fura-2/AM (Invitrogen), and intracellular $\mathrm{Ca}^{2+}$ transients were recorded with a dualexcitation fluorescence photomultiplier system (IonOptix). In brief, cells were bathed in extracellular solution containing (in $\mathrm{mM}$ ): 135 $\mathrm{NaCl}, 5.4 \mathrm{KCl}, 1.8 \mathrm{CaCl}_{2}, 1 \mathrm{MgCl}_{2}, 10$ glucose, 10 HEPES (pH 7.4). Myocytes were stimulated to contract at $1 \mathrm{~Hz}$. For $\mathrm{Ca}^{2+}$ transient peak measurements, autofluorescence and background emissions were first subtracted and the peak was measured as the difference from the baseline using IonWizard software (IonOptix) (51).

Echocardiography. Transthoracic echocardiography was performed on anesthetized mice using a VisualSonics Vevo 2100 high-resolution imaging system with a $30-\mathrm{mHz}$ imaging transducer. LAD was measured at end-systole in parasternal long axis view, and LV diastolic dimension (LVEDD) was measured at end-diastole in parasternal short axis views and modified 4 -chamber views. LVEF was measured in parasternal, modified 4-chamber and short axis views using Simpson's biplane formula.

Histology. Excised hearts were placed in $10 \%$ formalin and cut in coronal sections. Every other slice $(10 \mu \mathrm{m})$ was stained with hematoxylin-eosin and Masson Trichrome staining for fibrosis. Each slide was reviewed and photographed under light microscopy $\times 20$ to $\times 400$. Fibrosis was evaluated as a ratio of total blue pixels/total myocardial area in Masson's trichrome stained slices, using cellSens imaging software (Olympus). Two pathologists, who were blinded to the identity of the genotypes, independently reviewed the quantitative analyses.

TEM. The hearts were fixed with $2.5 \%$ glutaraldehyde in $0.1 \mathrm{M}$ Sorenson's buffer ( $\mathrm{pH}$ 7.2) and then postfixed with $1 \% \mathrm{OsO}_{4}$ in Sorenson's buffer for 1 hour. After dehydration, the hearts were embedded in Lx-112 (Ladd Research Industries). Thin sections (60 nm) were cut on a PT-XL ultramicrotome (Boeckeler Instruments). The sections were stained with uranyl acetate and lead citrate and examined under a JEOL JEM-1200 EXII electron microscope. Images were captured with an ORCA-HR digital camera (Hamamatsu) and recorded with an AMT Image Capture Engine. 
Epicardial optical voltage mapping of Langendorff-perfused hearts. High-resolution optical mapping experiments were performed on 4- to 12-month-old F1759A-dTG and littermate control mice. Hearts were isolated and perfused by the Langendorff method with temperature-controlled $\left(37^{\circ} \mathrm{C}\right)$ oxygenated Krebs-Henseleit buffer ( $\left.\mathrm{pH} 7.4\right)$. One $\mathrm{AgCl}$ wire was attached to the metal aortic cannula, and another $\mathrm{AgCl}$ wire was positioned near the surface of the heart to record a pseudo-ECG. Cardiac contraction was inhibited with $5 \mu \mathrm{M}$ blebbistatin. The right and left atria were splayed using tungsten pins. The heart was then stained with the voltage-sensitive dye Di4ANEPPS ( $8 \mu \mathrm{l}$ of $2 \mathrm{mM}$ stock solution dissolved in DMSO) and illuminated via epifluorescence. Fluorescence was acquired through a $715 \mathrm{~nm}$ pass filter using a CMOS camera (MICAM Ultima, SciMedia) with $1 \times 1 \mathrm{~cm}$ field of view. One- to 2-second recordings were captured at 1,000 $\mathrm{Hz}$. All optical signals were processed with custom MATLAB software downloaded from I. Efimov's laboratory website (Washington University, St Louis, Missouri, USA) as previously described $(52,53)$. DF was evaluated for each pixel as the frequency band with maximal power on a periodogram calculated with a fast Fourier transform. RI was defined as the ratio of the power within a $1-\mathrm{Hz}$ band centered on the $\mathrm{DF}$ and the total power spectrum from $0-100 \mathrm{~Hz}$ (52).

Statistics. Results are presented as mean \pm SEM. For comparisons between 2 groups, unpaired 2-tailed Student's $t$ test was used. Differences were considered statistically significant at $P<0.05$. All statistical analyses were performed with Prism 6.0.

Study approval. The Institutional Animal Care and Use Committee at Columbia University approved all animal experiments.

\section{Author contributions}

EW, JA, RLW, ANK, and SOM designed the study. EW, JA, RLW, ANK, SIZ, JB, LY, and JPM performed experiments and collected the data. EW, JA, RLW, ANK, SIZ, JB, LY, JPM, HG, and SOM analyzed the data. EW, JA, RLW, ANK, and SOM wrote the manuscript.

\section{Acknowledgments}

The authors thank I. Efimov, S. Gutbrod, and M. Sulkin (Washington University) for technical assistance on the optical imaging experiments and analysis; P. Nauleau for assistance with Matlab scripting; C. Marboe for pathology expertise; K. Brown for TEM image acquisition; and R. Kass and K. Sampson for technical insights concerning cellular electrophysiology. The research was supported by NHLBI R01 HL122967 and R01HL123483 to S.O. Marx, and a gift from the Bahna family to H. Garan. J. Abrams was supported by NHLBI F30 HL129628, R.L. Weinberg was supported by NHLBI T32HL007854, and J.P. Morrow was supported by NHLBI K08 HL105801. E. Wan was supported by the Louis V. Gerstner Jr. Scholars Program and the Esther Aboodi Endowed Professorship at Columbia University.

Address correspondence to: Steven O. Marx, Columbia University, College of Physicians and Surgeons, 622 W168th Street, PH-3 Center, New York, New York 10032, USA. Phone: 212.305.0271; E-mail:Sm460@cumc.columbia.edu.

Richard L. Weinberg's present address is: Division of Cardiovascular Medicine, Department of Internal Medicine, University of Michigan, Ann Arbor, Michigan, USA.
1. Heijman J, Voigt N, Nattel S, Dobrev D. Cellular and molecular electrophysiology of atrial fibrillation initiation, maintenance, and progression. Circ Res. 2014;114(9):1483-1499.

2. Vaziri SM, Larson MG, Benjamin EJ, Levy D. Echocardiographic predictors of nonrheumatic atrial fibrillation. The Framingham Heart Study. Circulation. 1994;89(2):724-730.

3. Wakili R, Voigt N, Kaab S, Dobrev D, Nattel $\mathrm{S}$. Recent advances in the molecular pathophysiology of atrial fibrillation. J Clin Invest. 2011;121(8):2955-2968.

4. Gudbjartsson DF, et al. A sequence variant in ZFHX3 on 16q22 associates with atrial fibrillation and ischemic stroke. Nat Genet. 2009;41(8):876-878.

5. Ellinor PT, et al. Meta-analysis identifies six new susceptibility loci for atrial fibrillation. Nat Genet. 2012;44(6):670-675.

6. Haissaguerre M, et al. Electrophysiological end point for catheter ablation of atrial fibrillation initiated from multiple pulmonary venous foci. Circulation. 2000;101(12):1409-1417.

7. Moss AJ, Kass RS. Long QT syndrome: from channels to cardiac arrhythmias. JClin Invest. 2005;115(8):2018-2024.

8. Toischer $\mathrm{K}$, et al. Role of late sodium current as a potential arrhythmogenic mechanism in the progression of pressure-induced heart disease. J Mol Cell Cardiol. 2013;61:111-122.

9. Darbar D, et al. Cardiac sodium channel (SCN5A) variants associated with atrial fibrillation. Circulation. 2008;117(15):1927-1935.

10. Johnson JN, Tester DJ, Perry J, Salisbury BA, Reed
CR, Ackerman MJ. Prevalence of early-onset atrial fibrillation in congenital long QT syndrome. Heart Rhythm. 2008;5(5):704-709.

11. Sossalla $\mathrm{S}$, et al. Altered $\mathrm{Na}(+)$ currents in atrial fibrillation effects of ranolazine on arrhythmias and contractility in human atrial myocardium. J Am Coll Cardiol. 2010;55(21):2330-2342.

12. Ragsdale DS, McPhee JC, Scheuer T, Catterall WA. Molecular determinants of state-dependent block of $\mathrm{Na}^{+}$channels by local anesthetics. Science. 1994;265(5179):1724-1728.

13. Remme CA, Bezzina CR. Sodium channel (dys) function and cardiac arrhythmias. Cardiovasc Ther. 2010;28(5):287-294.

14. Sanbe A, Gulick J, Hanks MC, Liang Q, Osinska H, Robbins J. Reengineering inducible cardiac-specific transgenesis with an attenuated myosin heavy chain promoter. Circ Res. 2003;92(6):609-616.

15. Valencik ML, McDonald JA. Codon optimization markedly improves doxycycline regulated gene expression in the mouse heart. Transgenic Res. 2001;10(3):269-275.

16. McNulty MM, Edgerton GB, Shah RD, Hanck DA, Fozzard HA, Lipkind GM. Charge at the lidocaine binding site residue Phe-1759 affects permeation in human cardiac voltage-gated sodium channels JPhysiol. 2007;581(pt 2):741-755.

17. Carboni M, Zhang ZS, Neplioueva V, Starmer $\mathrm{CF}$, Grant AO. Slow sodium channel inactivation and use-dependent block modulated by the same domain IV S6 residue. JMembr Biol. 2005;207(2):107-117.

18. Sossalla S, et al. Diastolic dysfunction and arrhythmias caused by overexpression of
CaMKIIdelta(C) can be reversed by inhibition of late $\mathrm{Na}(+)$ current. Basic Res Cardiol. 2011;106(2):263-272.

19. Maltsev VA, Sabbah HN, Higgins RS, Silverman N, Lesch M, Undrovinas AI. Novel, ultraslow inactivating sodium current in human ventricular cardiomyocytes. Circulation. 1998;98(23):2545-2552.

20. Valdivia CR, et al. Increased late sodium current in myocytes from a canine heart failure model and from failing human heart. J Mol Cell Cardiol. 2005;38(3):475-483.

21. Wagner E, et al. Stimulated emission depletion live-cell super-resolution imaging shows proliferative remodeling of T-tubule membrane structures after myocardial infarction. Circ Res. 2012;111(4):402-414.

22. Kohlhaas $\mathrm{M}$, et al. Elevated cytosolic $\mathrm{Na}^{+}$increases mitochondrial formation of reactive oxygen species in failing cardiac myocytes. Circulation. 2010;121(14):1606-1613.

23. Iwai $\mathrm{T}$, et al. Sodium accumulation during ischemia induces mitochondrial damage in perfused rat hearts. Cardiovasc Res. 2002;55(1):141-149.

24. Nuyens D, et al. Abrupt rate accelerations or premature beats cause life-threatening arrhythmias in mice with long-QT3 syndrome. Nat Med. 2001;7(9):1021-1027.

25. Riley G, Syeda F, Kirchhof P, Fabritz L. An introduction to murine models of atrial fibrillation. Front Physiol. 2012;3:296.

26. Lemoine MD, et al. Arrhythmogenic left atrial cellular electrophysiology in a murine genetic long QT syndrome model. Cardiovasc Res. 2011;92(1):67-74. 
27. Pandit SV, Jalife J. Rotors and the dynamics of cardiac fibrillation. Circ Res. 2013;112(5):849-862.

28. Martins RP, et al. Dominant frequency increase rate predicts transition from paroxysmal to longterm persistent atrial fibrillation. Circulation. 2014;129(14):1472-1482.

29. Dibs SR, Ng J, Arora R, Passman RS, Kadish AH, Goldberger JJ. Spatiotemporal characterization of atrial activation in persistent human atrial fibrillation: multisite electrogram analysis and surface electrocardiographic correlations - a pilot study. Heart Rhythm. 2008;5(5):686-693.

30. Ju YK, Saint DA, Gage PW. Hypoxia increases persistent sodium current in rat ventricular myocytes. J Physiol. 1996;497(pt 2):337-347.

31. Wagner S, et al. $\mathrm{Ca}^{2+} /$ calmodulin-dependent protein kinase II regulates cardiac $\mathrm{Na}^{+}$channels. J Clin Invest. 2006;116(12):3127-3138.

32. Luczak ED, Anderson ME. CaMKII oxidative activation and the pathogenesis of cardiac disease. J Mol Cell Cardiol. 2014;73:112-116.

33. Purohit A, et al. Oxidized $\mathrm{Ca}(2+) /$ calmodulindependent protein kinase II triggers atrial fibrillation. Circulation. 2013;128(16):1748-1757.

34. Scirica BM, et al. Effect of ranolazine, an antianginal agent with novel electrophysiological properties, on the incidence of arrhythmias in patients with non ST-segment elevation acute coronary syndrome: results from the Metabolic Efficiency With Ranolazine for Less Ischemia in Non STElevation Acute Coronary Syndrome Thrombolysis in Myocardial Infarction 36 (MERLIN-TIMI 36) randomized controlled trial. Circulation. 2007;116(15):1647-1652.

35. Bourgonje VJ, et al. Combined $\mathrm{Na}(+) / \mathrm{Ca}(2+)$ exchanger and L-type calcium channel block as a potential strategy to suppress arrhythmias and maintain ventricular function. Circ Arrhythm
Electrophysiol. 2013;6(2):371-379.

36. Milberg P, et al. Acute inhibition of the $\mathrm{Na}(+) /$ $\mathrm{Ca}(2+)$ exchanger reduces proarrhythmia in an experimental model of chronic heart failure. Heart Rhythm. 2012;9(4):570-578.

37. Mann SA, et al. R222Q SCN5A mutation is associated with reversible ventricular ectopy and dilated cardiomyopathy. J Am Coll Cardiol. 2012;60(16):1566-1573.

38. Davidenko JM, Kent PF, Chialvo DR, Michaels DC, Jalife J. Sustained vortex-like waves in normal isolated ventricular muscle. Proc Natl Acad Sci U S A. 1990;87(22):8785-8789.

39. Jalife J. Mechanisms of persistent atrial fibrillation. Curr Opin Cardiol. 2014;29(1):20-27.

40. Umapathy K, et al. Phase mapping of cardiac fibrillation. Circ Arrhythm Electrophysiol. 2010;3(1):105-114.

41. Dautova Y, Zhang Y, Sabir I, Grace AA, Huang CL. Atrial arrhythmogenesis in wild-type and Scn $5 \mathrm{a}+/ \Delta$ murine hearts modelling LQT3 syndrome. Pflugers Arch. 2009;458(3):443-457.

42. Guzadhur L, et al. Atrial arrhythmogenicity in aged Scn $5 \mathrm{a}+/ \Delta \mathrm{KPQ}$ mice modeling long $\mathrm{QT}$ type 3 syndrome and its relationship to $\mathrm{Na}^{+}$channel expression and cardiac conduction. Pflugers Arch. 2010;460(3):593-601.

43. Blana A, et al. Knock-in gain-of-function sodium channel mutation prolongs atrial action potentials and alters atrial vulnerability. Heart Rhythm. 2010;7(12):1862-1869.

44. Zhang T, et al. LQTS mutation N1325S in cardiac sodium channel gene SCN5A causes cardiomyocyte apoptosis, cardiac fibrosis and contractile dysfunction in mice. Int J Cardiol. 2011;147(2):239-245.

45. Zhang T, Yong SL, Tian XL, Wang QK. Cardiac-specific overexpression of SCN5A gene leads to shorter P wave duration and PR interval in transgenic mice. Biochem Biophys Res Commun. 2007;355(2):444-450

46. Gellens ME, et al. Primary structure and functional expression of the human cardiac tetrodotoxin-insensitive voltage-dependent sodium channel. Proc Natl Acad Sci U S A. 1992;89(2):554-558.

47. Wang C, Wang C, Hoch EG, Pitt GS. Identification of novel interaction sites that determine specificity between fibroblast growth factor homologous factors and voltage-gated sodium channels. J Biol Chem. 2011;286(27):24253-24263.

48. Hambleton M, et al. Inducible and myocytespecific inhibition of $\mathrm{PKC} \alpha$ enhances cardiac contractility and protects against infarctioninduced heart failure. Am J Physiol Heart Circ Physiol. 2007;293(6):H3768-H3771.

49. O'Connell TD, Rodrigo MC, Simpson PC. Isolation and culture of adult mouse cardiac myocytes. Methods Mol Biol. 2007;357:271-296.

50. Nguyen TP, Wang DW, Rhodes TH, George AL Jr. Divergent biophysical defects caused by mutant sodium channels in dilated cardiomyopathy with arrhythmia. Circ Res. 2008;102(3):364-371.

51. Hennessey JA, Wei EQ, Pitt GS. Fibroblast growth factor homologous factors modulate cardiac calcium channels. Circ Res. 2013;113(4):381-388.

52. Gutbrod SR, et al. Quantification of the transmural dynamics of atrial fibrillation by simultaneous endocardial and epicardial optical mapping in an acute sheep model. Circ Arrhythm Electrophysiol. 2015;8(2):456-465.

53. Laughner JI, Ng FS, Sulkin MS, Arthur RM, Efimov IR. Processing and analysis of cardiac optical mapping data obtained with potentiometric dyes. Am J Physiol Heart Circ Physiol. 2012;303(7):H753-H765. 